\title{
Syndrome $Z$ and Its Association with Obstructive Sleep Apnea
}

\author{
Gulam Hassan ${ }^{1}$, Waseem Qureshi ${ }^{2, *}$ \\ ${ }^{1}$ Department of Medicine, Government Medical College, Srinagar, India \\ ${ }^{2}$ Department of Chest Medicine/Registrar Academics, Government Medical College, Srinagar, India \\ Email address: \\ qureshiwaseem786@gmail.com(W.Qureshi) \\ ${ }^{*}$ Corresponding author
}

\section{To cite this article:}

Gulam Hassan, Waseem Qureshi. Syndrome Z and Its Association with Obstructive Sleep Apnea. International Journal of Diabetes and Endocrinology. Vol. 3, No. 1, 2018, pp. 15-17. doi: 10.11648/j.ijde.20180301.13

Received: February 26, 2018; Accepted: March 16, 2018; Published: May 3, 2018

\begin{abstract}
It is clear that obstructive sleep apnea (OSA) and metabolism syndrome share a similar pathophysilogic milieu that would be expected to increase the risk of cardiovascular disease. In patients with established coronary artery disease, treatment of OSA may confer long term cardiovascular benefits. Prevention of nocturnal hypoxemia, sympathetic activation and pressor surges in addition to reduction of daytime sympathetic activity, blood pressure and insulin resistance by continuous positive airway pressure would improve cardiovascular outcomes in patients with metabolic syndrome.
\end{abstract}

Keywords: Syndrome Z, Obstructive Sleep Apnea (OSA), Metabolic Syndrome

\section{Introduction}

Metabolic syndrome is characterized by the occurrence of metabolic risk factors for endothelial dysfunction and atherosclerotic cardiovascular disease that includes abdominal obesity, hyperglycemia, dyslipidemia and hypertension. Other names used for this spectrum of clinical and biochemical finding are syndrome $\mathrm{X}$, insulin resistance syndrome or obesity dyslpidemia syndrome, Reavan's syndrome or Kaplan's deadly quartet [1-3]. This syndrome is a co-occurrence or clustering of metabolic disturbances resulting in a higher risk of type 2 diabetes mellitus and cardiovascular disease, and may contribute to the pathogenesis of other complex diseases like colon and other cancers as well [4].

The metabolic syndrome has been defined by several authorities, including the World Health Organization [5], National Cholesterol Education Program Third Adult Treatment Panel (ATP-III) [6]., American Heart Association / National Heart, Lung and Blood Institute [2] and the International Diabetes Federation [7]. Despite some disparity among these definitions, all of these encompass some criteria of the four key elements: insulin resistance/glucose dysregulation, obesity, hypertension and dyslipidemia. The metabolic syndrome is being increasingly more prevalent worldwide; approximately 25 to $40 \%$ of adult Americans are reported to have this disorder [8-9].

As per the (NCEP-ATP) III criteria, metabolic syndrome is $40 \%$ greater in patients with obstructive sleep apnea (OSA), an illness characterized by repetitive episodes of partial (hypopnea) or complete (apnea) cessation of breathing during sleep. Though there is circumstantial evidence to implicate OSA in the development of metabolic syndrome, the causal relationship remains unproven. It has been hypothesized that in the setting of OSA and metabolic syndrome, there exists feed forward relationship between the two which leads to further aggravation of both disorders. It has been proposed that OSA may be one of the manifestations of metabolic syndrome $[10,11]$. There is published evidence suggesting independent association of OSA with insulin resistance and other components of metabolic syndrome [12]. The cooccurrence of OSA and metabolic syndrome is defined as Syndrome Z [13].

\section{Results}

OSA has been associated with all the four of the more established components of the metabolic syndrome, leading to the evolution of syndrome Z [14]. In a recent hierarchal 5factor model providing empirical evidence for syndrome $Z$, a 
good overall fit was found that obesity was the most important determining factor; followed by sleep disturbance, insulin resistance, hypertension and dyslipidemia [15].

There is a growing experimental and clinical evidence for an independent contribution of OSA toward the development and/or severity of individual metabolic disorders and the syndrome entity. On the other hand, metabolic syndrome and its components - in particular, obesity and insulin resistance / diabetes mellitus may have a conductive influence on the development of sleep apnea, it has also been proposed that OSA itself may be a metabolic disorder and a component of metabolic syndrome [16]. Several well designed studies support the association between OSA and metabolic syndrome in adults [16]. There is now ample evidence of independent association of OSA with systemic hypertension, insulin resistance, ischemic heart disease and stroke [18] as well. The mechanisms of underlying components of the syndrome $\mathrm{Z}$ are briefly described here.

\section{Discussion}

\subsection{Hypertension}

For hypertension to occur, endothelial dysfunction with decreased nitric oxide (No) production and subsequent predominance of vasoconstrictive mechanisms is considered to be one of the main factors [19]. More recently there has been evidence of synergistic or antagonistic effect of other substances on the arterial system - activation of arginase leading to decreased availability of L-arginine as an accessory factor of No synthase with subsequent fall in its production and decrease in its vasodilating effect [20]. Evidence also indicates that sodium reabsorption is increased in people with metabolic syndrome [21]. The mechanism by which OSA cuases hypertension is multifactorial, nocturnal sympathetic nervous system activation being the key factor.
In addition, sodium retension occurs in obesity and probably sleep apnea induced hypertension is associated with activation of the renin angiotensin - aldosterone system, and elevation of leptin levels. Hyperleptinemia may provide a broader link between OSA and hypertension because leptin may increase adrenergic activity per se, that is an important determinant of hypertension in OSA [22].

\subsection{Dyslipidemia}

In general, with increase in free fatty acid flux to the liver, increased production of apo-B-containing triglyceride-rich very low density lipoprotein (VLDL) takes place. In the setting of insulin resistance, increased flux of free fatty acids to the liver increases the hepatic triglyceride synthesis [23]. The other major lipoprotein disturbance in metabolic syndrome is a reduction in HDL cholesterol. In the presence of hypertriglyceridemia, a decrease in the cholesterol content of HDL results from decreases in the cholestryl ester content of the lipoprotein core with variable increase in triglyceride, a function in part of cholestryl ester transfer protein [24].

\subsection{Insulin Resistance and Diabetes Mellitus}

Insulin resistance is a precursor state of diabetes mellitus, and metabolic syndrome is also highly predictive of diabetes mellitus. OSA may also fuel other derangements attributable to insulin resistance, such as hypertension, hypertriglyceridemia, and visceral obesity, perpetuating the disturbances in metabolic syndrome and to its cardiovascular sequelae. OSA, insulin resistance and metabolic syndrome are closely related to indices of obesity [25]. There is enough published evidence to support the fact that there is increased risk of developing diabetes in individuals with short sleep durations and/or difficulties sleeping at baseline. It is likely that multiple interrelated factors contribute to the complex interaction between OSA, obesity and glucose metabolism (Figure 1).

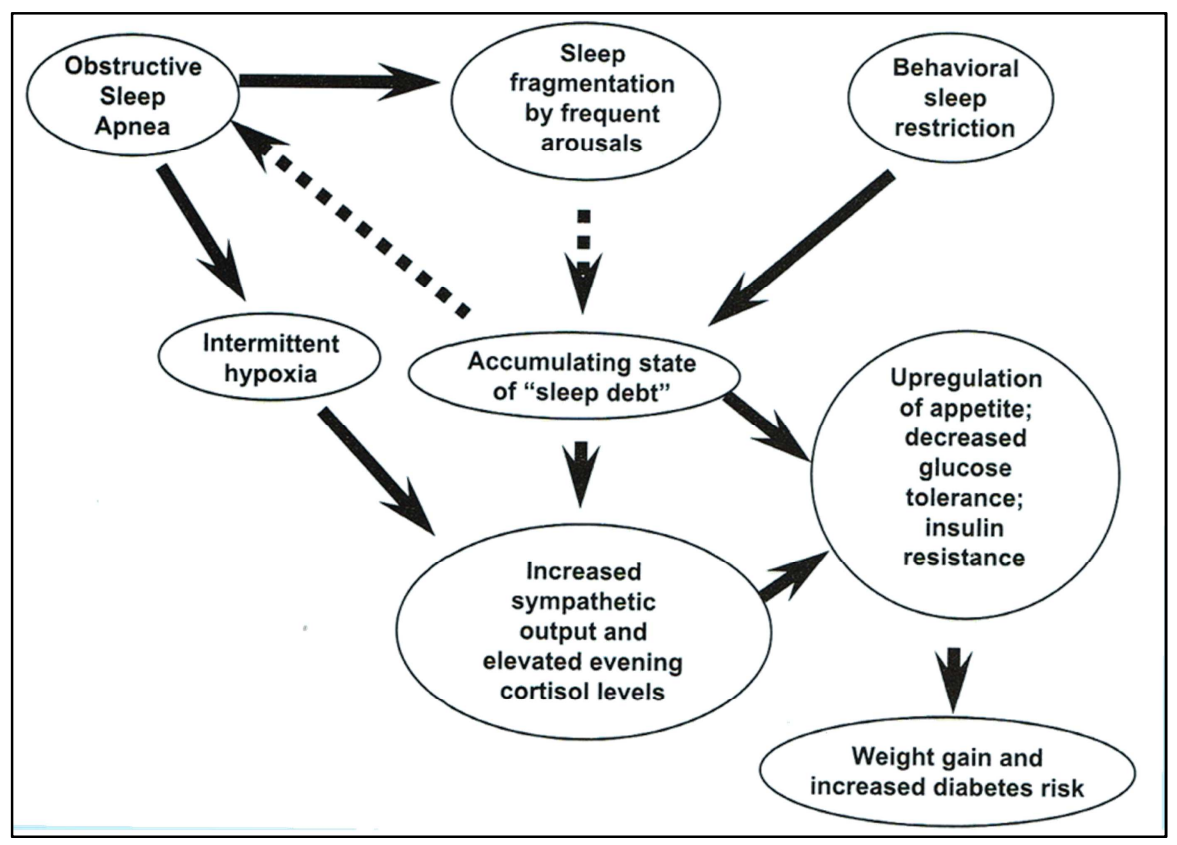

Figure 1. Mechanisms leading to insulin resistance and increased diabetes risk in obstructive sleep apnea. 
OSA is associated with chronic intermittent hypoxia and sleep fragmentation, which may adversely affect glucose homeostasis. Increased sympathetic activity, dysregulation of hypothalamus - pituitary axis, generation of reactive oxygen species, and activation of inflammatory pathways have all been proposed as causative stimuli that lead to alteration in glucose metabolism in OSA [17]. Studies have shown a supportive evidence of a dose dependent relationship between the severity of OSA and glucose metabolism [26].

\section{Conclusion}

It is clear that OSA and metabolism syndrome share a similar pathophysilogic milieu that would be expected to increase the risk of cardiovascular disease. In patients with established coronary artery disease, treatment of OSA may confer long term cardiovascular benefits.

\section{References}

[1] Eckel RH, Grundy SM, Zimmet PZ. The metabolic syndrome. Lancet 2005; 365: 1415-28.

[2] Grundy SM, Brewer HB, Cleeman Ji, Smith SC, Lenfant C. Definition of metabolic syndrome: report of the National Heart, Lung and Blood Institute / American Heart Association Conference on scientific issues related to definition. Circulation 2004; 109: 433-8.

[3] Raven GM. Role of insulin resistance in human disease. Diabetes 1988; 37: 1595-1607.

[4] Coweys S. Hardy W. The metabolic syndrome: a high risk state for cancer? Am J Pathol 2004; 109: 433-8.

[5] Alberti KG, Zimmet PZ. Definition, diagnosis and classification of diabetes mellitus and its complications. Part 1: diagnosis and classification of diabetes mellitus provisional report of a WHO consultation. Diabet Med 1998; 15: 539-53.

[6] Third Report of the National Cholesterol Education Program (NCEP) Expert Panel on Detection, Evaluation and Treatment of High Blood Cholesterol in Adults (Adult Treatment Panel III) final report. Circulation 2002; 106: 3143-421.

[7] International Diabetes Federation. The IDF Consensus Worldwide. Definition of the metabolic syndrome. http://www.idf.org/ webdata/docs/IDF_Metadef_final.pdf2006: 1-14.

[8] Milani RV, Lavie CJ. Prevalence and profile of metabolic syndrome in patients following acute coronary events and effects of therapeutic life style change with cardiac rehabilitation. Am J Cardiol 2003; 92: 50-4.

[9] Ford ES. Prevalence of metabolic syndrome defined by the International Diabetes Federation among adults in the U.S. Diabetes Care 2005; 28: 2745-9.

[10] Coughlin SR, Mawdsley L, Mugarza JA, Calverley P, Wilding JP. Obstructive sleep apnea is independently associated with an increased prevalence of metabolic syndrome. Eur Heart J 2004; 25: 735-41.
[11] Vgontzas AN, Bixler EO, Chrousos GP. Sleep apnea is a manifestation of the metabolic syndrome. Sleep Med Rev 2005; 9: 211-24.

[12] Ip MS, Lam B, Ng MM, Lam WK, Tsang KW, Lan KS. Obstructive sleep apnea is independently associated with insulin resistance. Am J Respir Crit Care Med 2002; 165: 6706.

[13] Wilcox I, Mc Namara SG, Collins FL, Grunstein RR, Sullivan CE. "Syndrome Z": the interaction of sleep apnoea, vascular risk factors and heart disease. Thorax 1998; 53 (Suppl. 3): S25-8.

[14] Young T, Peppard PE, Gottlieb DJ. Epidemiology of obstructive sleep apnea: a population health perspective. Am J Respir Crit Care Med 2002; 165: 1217-39.

[15] Nock NL, Li Li, Larkin EK, Patel SR, Redline S. Empirical evidence for "Syndrome Z": a hierarchial 5-factor model of the metabolic syndrome incorporating sleep disturbance measures. Sleep 2009; 32: 615-22.

[16] Vgontzas AN, Bixler EO, Chrousos GP. Sleep apnea is a manifestation of metabolic syndrome. Sleep Med Rev 2005; 9: 211-24.

[17] Tasali E, Ip MSM. Obstructive sleep apnea and metabolic syndrome: alterations in glucose metabolism and inflammation. Proc Am Thorac Soc 2008; 5: 207-17.

[18] Sahar E, Whitney CN, Redline S et al. Sleep disordered breathing and cardiovascular disease: cross-sectional results of the Sleep Heart Health Study. Am J Respir Crit Care Med 2001; 163: 19-25.

[19] Nagota T, Kuo L, Ren Y, Yoshida A, Hein TW. C-reactive protein inhibits endothelium-dependent nitric oxide-mediated dilation of retinal arterioles via enhanced superoxide production. Invest Ophthalmol Vis Sci 2008; 49: 2053-60.

[20] Santhenam L, Chistianson DW, Nythan D, Berkowitz DE. Arginase and vascular aging. J Apple Physiol 2008; 105: 1632-42.

[21] Barhato A, Cappuccio FP, Folkerd EJ et al. Metabolic syndrome and renal sodium handling in three ethnic groups living in England. Diabetologia 2004; 47: 40-46.

[22] Pratt Ubunama MN, Nishizaka MK, Boedefeld RL et al. Plasma aldolesterone is related to severity of obstructive sleep apnea in subjects with resistant hypertensive. Chest 2007; 131: 453-59.

[23] Lewis GF, Uffelman KD, Szeto LW, Weller B, Steiner G. Interaction between free fatty acids and insulin in the acute control of very low density lipoprotein production in humans. J Clin Invest 1995; 95: 158-66.

[24] Murakami T, Michelgnoli S, Longhi R et al. Triglycerides are major determinants of cholesterol esterification/transfer and HDL remodeling in human plasma. Arterioscler Thromb Vasc Biol 1995; 15: 1819-28.

[25] Young T, Sahar E, Nieto FJ et al. Predictors of sleepdisordered breathing in community-dwelling adults: the Sleep Heart Health Study. Arch Intern Med 2002; 162: 893-900.

[26] Tamura T, Sahar E, Nieto FJ et al. Relationship between the severity of obstructive sleep apnea and impaired glucose metabolism in patients with obstructive sleep apnea. Respir Med 2008; 102: 1412-6. 